\title{
Wahlverhalten bei Landtagswahlen: Gute Basis für weitere Forschung
}

Völkl, Kerstin: Reine Landtagswahlen oder regionale Bundestagswahlen? Eine Untersuchung des Abstimmungsverhaltens bei Landtagswahlen 1990-2006 (Studien zur Wahl- und Einstellungsforschung, Band 14), Nomos Verlagsgesellschaft, Baden-Baden 2009, 295 Seiten, $€ 39$,-

Die Landesebene ist der in Deutschland dominierenden Bundesebene politisch nachgeordnet. Landtagswahlen werden daher häufig als Wahlen zweiter Ordnung bezeichnet und befinden sich auch nicht im zentralen Fokus der deutschen Wahlforschung. Obwohl in den letzten Jahren das Interesse an ihnen gestiegen ist, gelten sie immer noch als weitgehend vernachlässigtes Gebiet der Disziplin. Analysen zu Landtagswahlen sind in der Regel deskriptiver Natur und lassen verallgemeinerbare Schlüsse vermissen. Eine zentrale Frage bei der Beschäftigung mit Landtagswahlen ist die nach der Bedeutung der Bundespolitik für die individuelle Wahlentscheidung. An dieser Stelle setzt Kerstin Völkl mit ihrer Dissertation an.

Nach einer kurzen Darstellung des Forschungsproblems geht die Autorin auf den Forschungsstand ein. Dabei berücksichtigt sie sowohl die nationalen als auch die im Zusammenhang mit Landtagswahlen häufig vernachlässigten internationalen Veröffentlichungen der Forschung zu Wahlen in Mehrebenensystemen. Sie verweist auf Probleme bei der Übertragbarkeit der vorwiegend aus den USA stammenden Ansätze beispielsweise zu Zyklustheorien. Des Weiteren stellt sie die heterogenen Ergebnisse zum Bundeseinfluss auf das Landtagswahlverhalten dar, die sowohl dessen Abnehmen als auch seine Zunahme über die letzten Jahre attestieren.

Anschließend entwickelt Völkl ausgehend vom klassischen Michigan-Ansatz ein Modell für die Wahlentscheidung bei Landtagswahlen. In der Modellierung sind bundes-, landesund ebenenunspezifische Einflüsse auf das Wahlverhalten enthalten. Bedauerlicherweise kommt dieses Modell als Konsequenz aus der schlechten Datenlage für die spätere Analyse nur stark reduziert zur Anwendung. Entsprechend der Modellierung formuliert Völkl eine Reihe von Hypothesen über die Wirkung von Parteiidentifikation, Regierungskonstellationen in Bund und Land, die Bedeutung von Spitzenpolitikern, aber auch den Zeitpunkt von Landtagswahlen im Bundeszyklus.

Bevor die empirische Analyse der individuellen Entscheidung bei Landtagswahlen unter besonderer Berücksichtigung des Bundeseinflusses durchgeführt wird, beschäftigt sich Völkl ausführlich mit den möglichen kontextuellen Determinanten des Landtagswahlverhaltens. Dabei geht sie deskriptiv vor und berücksichtigt politische und wirtschaftliche Strukturund Konjunkturindikatoren auf Landes- und Bundesebene. Darunter fallen auch aggregierte Einstellungen zu Politikern, Parteien und Regierungen sowie die Einschätzungen der wichtigsten Probleme.

Die empirische Analyse basiert auf einem in der bisherigen Landtagswahlforschung üblichen Verfahren. Für die Wahlentscheidung zu Gunsten von Union und SPD werden binäre logistische Regressionen durchgeführt. Für jede dieser Wahlentscheidungen werden Bundes-, Landes- und Gesamtmodell berechnet, die eine Einstufung als „reine Landtagswahl“, „kleine Bundestagswahl“ oder „Mischwahl“ ermöglichen, je nachdem welche Beiträge die Einstellungen zu einer Ebene zum Modellfit beitragen. Dabei werden die meisten Landtagswahlen dem Typ der Mischwahl zugeordnet. 
Von den zentralen Ergebnissen der Untersuchung sind der nicht nachweisbare Einfluss des Zeitpunktes einer Landtagswahl im Bundeszyklus sowie die Bedeutung der Regierungskonstellationen in Bund und Land hervorzuheben. Für die Wahlentscheidung zu Gunsten einer Partei, die an der Landesregierung beteiligt ist, ist der Bundeseinfluss deutlich geringer als für Parteien, die an der Bundesregierung oder keiner Regierung beteiligt sind. Neben vielen weiteren Ergebnissen zeigt Völkl auch die dominante und stabile Wirkung der Parteiidentifikation für das Wahlverhalten, die vor dem Hintergrund der unklaren Ebenenzuordnung problematisch für die Einschätzung des Übergewichts einer der Ebenen ist. Insgesamt werden aber auch bisherige Einschätzungen zur großen Variation der Befunde über die Länder und Wahlen bestätigt.

Die Arbeit endet mit einem Ausblick auf einige offene Fragen und Probleme im Zusammenhang mit der Analyse von Landtagswahlen. Dazu gehören die bereits genannte ungeklärte Stellung bezüglich der Ebenenzuordnung der Parteiidentifikation, die Bedeutung von Kontextmerkmalen für das individuelle Wahlverhalten bei Landtagswahlen, die Entstehung bundes- und landespolitischer Einstellungen und nicht zuletzt das Datendefizit, das die meisten offenen Fragen für die vergangenen Wahlen unbeantwortbar lässt und auch einer größeren vergleichenden Analyse auf Jahre im Wege stehen wird.

Völkls Dissertation bietet eine umfassende Darstellung des Forschungsstandes und erschließt das individuelle Wahlverhalten in systematischer Weise. Dabei greift „Reine Landtagswahlen oder regionale Bundestagswahlen?“ auf denselben Datenbestand wie der von Völkl und anderen herausgegebene Band „Wähler und Landtagswahlen in der Bundesrepublik Deutschland“ zurück. Die Arbeit deckt einen kürzeren Zeitraum ab, und die Modellierung des Wahlverhaltens ist weniger originell und anregend als die des genannten Sammelbandes. Ihr Beitrag besteht in der stringenten Anwendung eines Analysemodells auf alle Landtagswahlen im untersuchten Zeitraum. Dadurch geht ihre Dissertation über bloße Länderstudien hinaus. Grundlegende Modellierungsprobleme wie die Konfundierung der wahlbeeinflussenden Variablen auf Bundes- und Landesebene konnten dabei nicht aufgelöst werden, was die Ergebnisse unter Vorbehalt stellt. Die Einteilung von Wahlen in „reine Landtagswahl“, „kleine Bundestagswahl“ oder „Mischwahl“ ist ungünstig gewählt, da sie die mögliche bundespolitische Durchdringung zu holzschnittartig erfasst. Zudem finden sich durch die gewählte Vorgehensweise zu viele Mischwahlen bei der Einstufung, was zwar der Realität entsprechen mag, aber auch eine Schwäche der Typologie aufzeigt.

Eine systematisch vergleichende Längsschnittuntersuchung des Bundeseinflusses auf Landtagswahlverhalten konfrontiert Politikwissenschaftler stets mit einem Dilemma. Es können entweder spezielle Modelle für jede Wahl berechnet werden, die kaum vergleichbare Ergebnisse liefern oder übersimplifizierte, die zwar vergleichbar sind, die zuvor gemachten theoretischen Überlegungen aber nur im Ansatz widerspiegeln. Völkl ist sich dieser Problematik bewusst, macht sie transparent und entscheidet sich für die Vergleichbarkeit. Ihre Analysen entsprechen dem Forschungsstand, resultieren nicht immer in spektakulären Ergebnissen, bilden jedoch eine gute Basis für weitere Forschung mit hoffentlich angemesseneren Daten. 